\title{
Integrated fiber-optic coupler for very large scale integration interconnects
}

\author{
Paul R. Prucnal, Eric R. Fossum, and Richard M. Osgood \\ Department of Electrical Engineering, Columbia University, New York, New York 10027
}

Received August 16, 1985; accepted November 14, 1985

A new optical interconnect technique, suitable for high packing densities, is demonstrated for implementation in very-large-scale integration circuits. The approach permits vertical bonding.

The performance of very-high-speed integrated circuits and very-large-scale integrated circuits (VLSIC's) is impaired by limitations in the communication capacity among gates, chip, and boards. ${ }^{1} \mathrm{Fi}-$ ber-optic interconnects are widely recognized as a potential solution to this communication problem. ${ }^{2-7}$ However, no satisfactory means exists to couple a fiber to a detector on a VLSIC chip. We demonstrate a new optical interconnection technique that, relative to previous methods, offers several advantages such as high packing density, accurate alignment, and mechanically stable coupling.

The advantages of optical over electronic interconnections include increased transmission bandwidth, immunity to mutual interference, freedom from capacitive loading, and freedom from planar constraints. ${ }^{5}$ The large information capacity of optical fibers can relieve the electronic interconnection bottleneck known as the "pinout problem." Optical interconnections also have the potential for reconfigurable switching and optically controlled electronic logic. ${ }^{3}$

Standard fiber-coupling techniques such as butt coupling or silicon V groove are not suitable for VLSIC interconnections. Butt coupling may be mechanically unstable, provide poor alignment, and not be suitable for high packing densities. V-groove coupling is not easily aligned with a detector array on a chip, and there are problems associated with multigroove splicing. ${ }^{8}$

The new optical interconnect technique provides excellent alignment and mechanical stability, requires minimal area on the surface of the chip (approximately $100 \mu \mathrm{m}^{2}$ ), and is well suited for high packing densities. Furthermore, interconnections are not confined to the perimeter of the chip but can be located at any point on the chip surface. The basic idea (see Fig. 1) is to etch a cylindrical hole, slightly larger than a singlemode optical-fiber core, into a semiconductor wafer. A $p-n$ junction, which serves as a detector, is then formed on the inside of the hole. A single-mode fiber core is inserted into the hole and affixed using epoxy or a simple mechanical fit. Light emanating from the fiber core is collected by the reverse biased $\mathrm{p}-\mathrm{n}$ junction and converted into an electrical signal. This signal is utilized by the circuitry on the surface of the wafer.
The seat for the optical fiber is fabricated by using laser-assisted etching of deep, high-aspect wells in the silicon substrate. The technique, which has been described elsewhere, ${ }^{9}$ consists of using the $257-\mathrm{nm}$ light from a frequency-doubled argon-ion laser to accomplish low-temperature, light-assisted etching in a $5 \%$ aqueous solution of $\mathrm{HF}$. In this process, the hole diameter is controlled to first order by the diameter of the nearly focused beam at the surface of the substrate. The diameter of the well used in this case is 12 $\mu \mathrm{m}$. A limited variation about this diameter can be effected by changing the beam power. Wells as narrow as $4 \mu \mathrm{m}$ have been made in the course of this project. Figure 2 shows the resulting cross section of a typical etched fiber well. Note that the side walls of the etch are nearly vertical. This is a result of light guiding during the etching process. In general, the well walls are smooth and nearly circular. This ensures good mechanical and optical coupling to the silicon substrate.

Following the etching of the well, the detector is made using standard semiconductor-device fabrication techniques. The well is doped using a spin-on

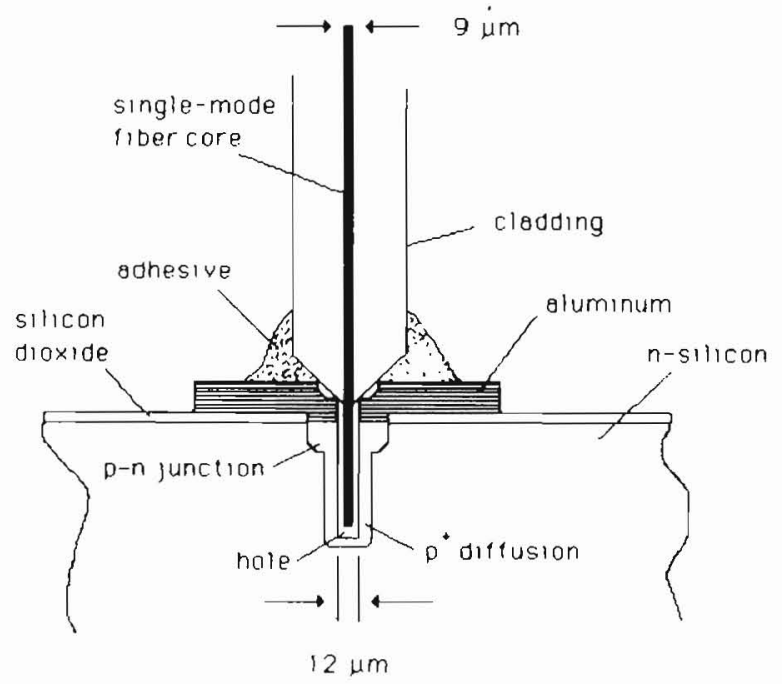

Fig. 1. Schematic cross section of prototype integrated fiber-optic coupler (IFOC) device. 


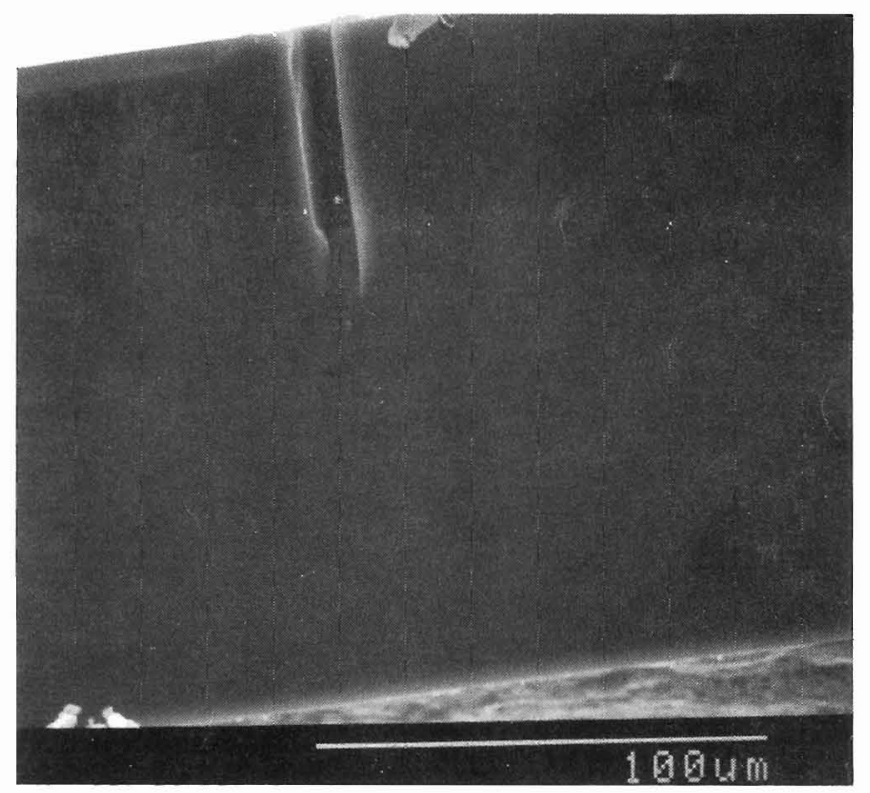

Fig. 2. Cross section of hole etched in silicon by a laserassisted photochemical progress. Hole is $50 \mu \mathrm{m}$ deep and 12 $\mu \mathrm{m}$ in diameter, illustrating viable high-aspect-ratio hole etching technology. (Etching and cleaving, P. V. Podlesnik; scanning electron microscope photo, $\mathrm{S}$. Todorov.)

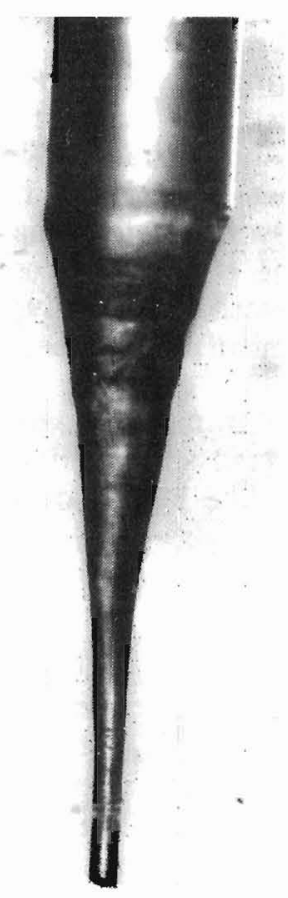

Fig. 3. Etched fiber at $150 \times$ magnification.

dopant film followed by a high-temperature drive-in. Although the formation of a $\mathrm{p}-\mathrm{n}$ junction is evident from the detector's current-voltage characteristics, the details of junction formation in the cavity are still under investigation. For metallization, aluminum is thermally evaporated and then delineated using a wet etch. A backside aluminum contact is also deposited.

A single-mode optical fiber is then etched and inserted into the detector cavity. The fiber is chemical- ly etched down to its $9-\mu \mathrm{m}$-diameter core by immersion in a continuously stirred solution of buffered HF for approximately $2 \mathrm{~h}$. (see Fig. 3 ). Only the tip of the fiber, from which the plastic coating is removed, is etched. The etched fiber is then inserted into the detector well by using the setup shown in Fig. 4.

The performance of the completed optical interconnect is measured by injecting light into the fiber and measuring the photocurrent generated on the silicon chip. The light from a cw $\mathrm{He}-\mathrm{Ne}$ laser, operating at $\lambda=0.63 \mu \mathrm{m}$, is focused on the cleaved unetched fiber end using a microscope objective. The output from the etched fiber end is measured to be approximately 1 $\mathrm{mW}$, using an integrating sphere. After insertion of the etched fiber end into the hole, the $I_{V}$ characteristic of the photodiode is measured using a curve tracer. The $I_{V}$ characteristic is shown in Fig. 5, under the conditions of no illumination (upper curve) and illumination from the tapered fiber end (lower curve).

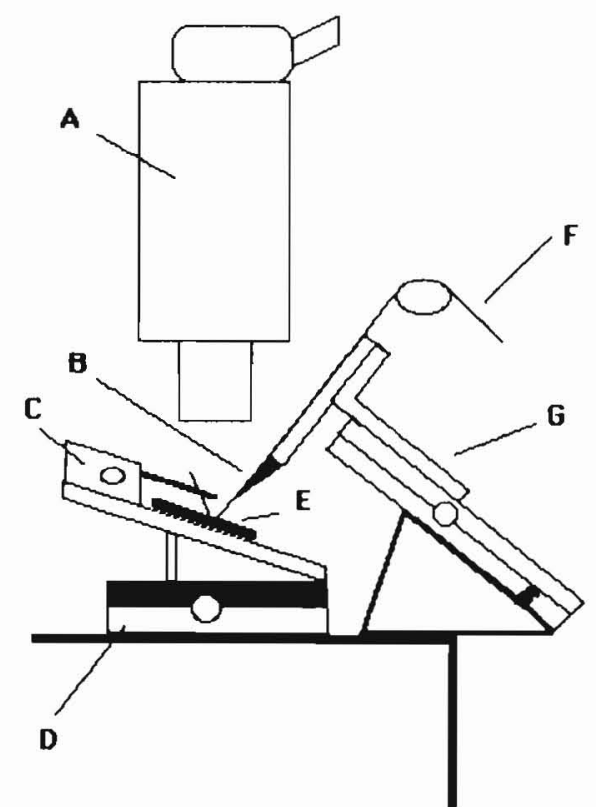

Fig. 4. Fiber insertion station: A, stereo microscope; B, vacuum fiber holder; $\mathrm{C}$, contact probe; $\mathrm{D}, X-Y$ positioner; $\mathrm{E}$, sample; $\mathrm{F}$, optical fiber; $\mathrm{G}, X-Y-Z$ positioner.

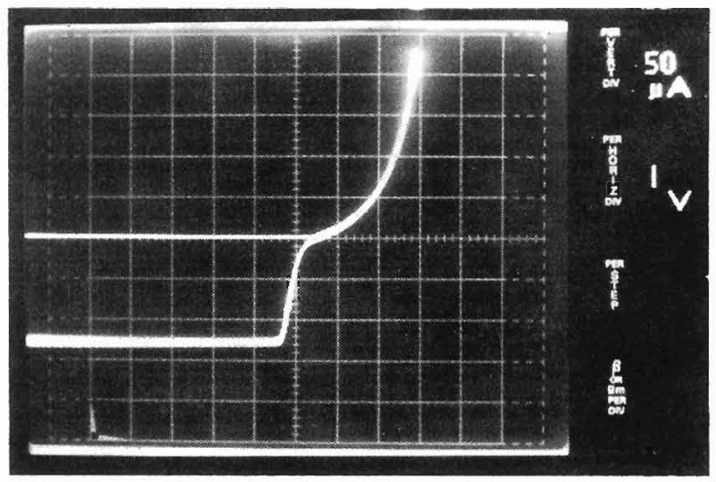

Fig. 5. Dark and illuminated $I_{V}$ characteristic of prototype IFOC device. 
The downward shift of the $I_{V}$ characteristic under illumination corresponds to a photon-generated current of $0.13 \mathrm{~mA}$. If all the light leaving the fiber is collected by the photodiode, the responsivity of the photodiode is approximately $0.13 \mathrm{~A} / \mathrm{W}$, corresponding to a quantum efficiency of approximately $25 \%$. Such efficiency is reasonable for a nonoptimized $\mathrm{p}-\mathrm{n}$ junction diode. In fact the quantum efficiency is somewhat larger than this, since some of the light leaving the fiber does not impinge upon the photodiode. With improvements in processing techniques for detector fabrication and single-mode fiber etching, it is expected that higher efficiency and less series resistance will be obtained.

Though the technique described here has been experimentally tested with silicon circuits, the same procedure can be applied to GaAs integrated circuits. In principle, such a vertical structure can then be applied to achieve a transmitter geometry as well.
The support of Defense Advanced Research Projects Agency contract no. N66001-85-C-0158 is gratefully acknowledged, as is the technical assistance of Amirhassan Amirfazli, Ted Cacouris, and Rick Colbeth.

\section{References}

1. R. W. Keyes, Proc. IEEE 63, 740 (1975).

2. A. Husain, Proc. Soc. Photo-Opt. Instrum. Eng. 466, 10 (1984)

3. J. W. Goodman, F. I. Leonberger, S. Y. Kung, and R. A. Athale, Proc. IEEE 72, 850 (1984).

4. A. Husain, Photo. Spectra 18(8), 57 (1984).

5. J. W. Goodman, Opt. Eng. Rep. (November 1984), p. 5.

6. J. A. Neff, Opt. Eng. 24, 1 (1985).

7. J. A. Neff, Opt. Eng. Rep. (April 1985), p. 1.

8. Y. Ando and I. Nishi, Appl. Opt. 21, 2689 (1982).

9. D. V. Podlesnik, H. H. Gilgen, and R. M. Osgood, Jr., Appl. Phys. Lett. 45, 563 (1984). 